\title{
An extension of Lagrange interpolation to approximate derivative, integral and bivariate function
}

\author{
Loc Nguyen \\ Institute of Mathematics, Hanoi, Vietnam \\ Email:ng_phloc@yahoo.com \\ Homepage: www.locnguyen.net
}

\begin{abstract}
Lagrange interpolation is the effective method to approximate an arbitrary function by a polynomial. But there is a need to estimate derivative and integral given a set of points. Although it is possible to make Lagrange interpolation first, which produces Lagrange polynomial; after that we take derivative or integral on such polynomial. However this approach does not result out the best estimation of derivative and integral. This research proposes a different approach that makes approximation of derivative and integral based on point data first, which in turn applies Lagrange interpolation into the approximation. Moreover, the research also proposes an extension of Lagrange interpolation to bivariate function, in which interpolation polynomial is converted as two-variable polynomial.
\end{abstract}

Keywords: Lagrange interpolation, polynomial, approximation.

\section{Lagrange interpolation of derivative and integral}

Given univariate function $f: \mathbb{R} \rightarrow \mathbb{R}$ where $\mathbb{R}$ is real number field, let $P(x)$ be interpolation polynomial [Wikipedia 2014] of $f$. Suppose $D=\left\{\left(x_{i}, f\left(x_{i}\right)\right\}\right.$ is the experimental data to estimate the polynomial $P(x)$ where all $x_{i}$ (s) are arranged in ascending order such as $x_{0} \leq$ $x_{1} \leq \cdots \leq x_{n}$ and so $x_{i}$ (s) form a partition whose elements $\left[x_{i}, x_{i+1}\right]$ are called volumes. In one-dimension space like $\mathbb{R}$, each volume is real number interval. According to Lagrange interpolation [Ta 2010] [Chapra, Canale 2010], we have:

$$
P(x)=\sum_{i=0}^{n}\left(f\left(x_{i}\right) \prod_{\substack{j=0 \\ j \neq i}}^{n} \frac{x-x_{j}}{x_{i}-x_{j}}\right)
$$

Let $P_{i}(x)=\prod_{\substack{j=0 \\ j \neq i}}^{n} \frac{x-x_{j}}{x_{i}-x_{j}}$ be the indicator function. Obviously, we have

$$
P(x)=\sum_{i=0}^{n} f\left(x_{i}\right) P_{i}(x)
$$

And,

$$
P_{i}\left(x_{j}\right)=\delta_{i j}=\left\{\begin{array}{l}
1 \text { if } i=j \\
0 \text { if } i \neq j
\end{array}\right.
$$

It is necessary to modify Lagrange method for interpolating derivative and integral. Let $\frac{\Delta f}{\Delta x}$ be the approximation of the derivative of function $f$. The derivative approximation at $x_{i}$ denoted $\frac{\Delta f}{\Delta x}\left(x_{i}\right)$ is computed as following:

$$
\frac{\Delta f}{\Delta x}\left(x_{i}\right)=\frac{f\left(x_{i+1}\right)-f\left(x_{i}\right)}{x_{i+1}-x_{i}}
$$

Let $\frac{\Delta P}{\Delta x}$ be the interpolation polynomial of derivative of function $f$, we have: 


$$
\frac{\Delta P}{\Delta x}=\sum_{i=0}^{n-1} \frac{\Delta f}{\Delta x}\left(x_{i}\right) P_{i}(x)=\sum_{i=0}^{n-1}\left(\frac{f\left(x_{i+1}\right)-f\left(x_{i}\right)}{x_{i+1}-x_{i}} \prod_{\substack{j=0 \\ j \neq i}}^{n-1} \frac{x-x_{j}}{x_{i}-x_{j}}\right)
$$

The ideology of interpolation of derivative is to make the discrete approximation of derivatives first, and then, to make interpolation on such approximations later. The interpolation of integral is computed by the similar way. Let $s(x)$ be the approximation of the integral of function $f$. The integral approximation at $x_{i}$ denoted $s\left(x_{i}\right)$ is computed as follows:

Where,

$$
s\left(x_{i}\right)=\frac{1}{2} \sum_{j=0}^{i-1}\left(f\left(x_{j}\right)+f\left(x_{j+1}\right)\right)\left(x_{j+1}-x_{j}\right)
$$

$$
s\left(x_{0}\right)=0 \text { and } 1 \leq i \leq n
$$

Essentially, $s\left(x_{i}\right)$ is the sum of areas of all trapeziums on the partition $x_{0} \leq x_{1} \leq \cdots \leq x_{i}$. We recognize that $s\left(x_{i}\right)$ is an accumulated function and so we should take advantage of its recursion so as to get high performance in iterative algorithm.

$$
s\left(x_{i}\right)=s\left(x_{i-1}\right)+\frac{1}{2}\left(f\left(x_{i-1}\right)+f\left(x_{i}\right)\right)\left(x_{i}-x_{i-1}\right)
$$

Let $S(x)$ be the interpolation polynomial of integral of function $f$, we have:

$$
S(x)=\sum_{i=0}^{n} s\left(x_{i}\right) P_{i}(x)=\sum_{i=0}^{n}\left(s\left(x_{i}\right) \prod_{\substack{j=0 \\ j \neq i}}^{n} \frac{x-x_{j}}{x_{i}-x_{j}}\right)
$$

The interpolation polynomial is deviated from the real integral of $f$ a constants $C$ with attention that the interpolation operation starts with the value $x_{1}$. The fact $s\left(x_{0}\right)=0$ implies that $S(x)$ is pulled back to the coordinate origin $(0,0)$ a constant $C$ according to $f(x)$-axis. Hence, $S(x)$ needs pushed away from the origin a distance $C$. Let $A(x)$ be the adjusted interpolation polynomial, we have:

$$
A(x)=S(x)+C
$$

The constant $C$ is calculated as the area of triangle formed by three points $(0,0),\left(x_{0}, 0\right)$ and $\left(x_{0}, f\left(x_{0}\right)\right)$.

$$
C=\frac{1}{2} f\left(x_{0}\right) x_{0}
$$

The adjusted interpolation polynomial $A(x)$ are totally determined as follows:

$$
A(x)=S(x)+\frac{1}{2} f\left(x_{0}\right) x_{0}
$$

\section{Interpolation of bivariate function}

When $f$ becomes bivariate function as $f: \mathbb{R}^{2} \rightarrow \mathbb{R}$, it is necessary to extend Lagrange interpolation. In this case, the experimental data becomes multivariate data $D=\left\{\left(x_{i}, y_{j}, f\left(x_{i}\right.\right.\right.$, $\left.\left.y_{j}\right)\right\}$ whose partition is $\left\{\left[x_{i}, x_{i+1}\right] \times\left[y_{j}, y_{j+1}\right]\right\}$ such that $x_{0} \leq x_{1} \leq \cdots \leq x_{m}$ and $y_{0} \leq y_{1} \leq$ $\cdots \leq y_{n}$. Note that volumes $\left[x_{i}, x_{i+1}\right] \times\left[y_{j}, y_{j+1}\right]$ are rectangles. The indicator function $P_{i j}(x, y)$ is re-defined as follows: 


$$
P_{i j}(x, y)=\prod_{\substack{k=0 \\ k \neq i}}^{m}\left(\frac{x-x_{k}}{x_{i}-x_{k}} \prod_{\substack{l=0 \\ l \neq j}}^{n} \frac{y-y_{l}}{y_{j}-y_{l}}\right)=\prod_{\substack{k=0 \\ k \neq i}}^{m} \prod_{\substack{l=0 \\ l \neq j}}^{n} \frac{\left(x-x_{k}\right)\left(y-y_{l}\right)}{\left(x_{i}-x_{k}\right)\left(y_{j}-y_{l}\right)}
$$

It is easy to infer that $P_{i j}(x, y)$ is multi-target indicator function.

$$
P_{i j}\left(x_{k}, y_{l}\right)=\delta_{i j k l}=\left\{\begin{array}{c}
1 \text { if } i=k \text { and } j=l \text { and } f\left(x_{k}, y_{l}\right) \text { exists } \\
0 \text { otherwise }
\end{array}\right.
$$

The bivariate interpolation polynomial $P(x, y)$ is expended as following:

$$
P(x, y)=\sum_{i=0}^{m} \sum_{j=0}^{n} f\left(x_{i}, y_{i}\right) P_{i j}(x, y)=\sum_{i=0}^{m} \sum_{j=0}^{n}\left(f\left(x_{i}, y_{i}\right) \prod_{\substack{k=0 \\ k \neq i}}^{m} \prod_{\substack{l=0 \\ l \neq j}}^{n} \frac{\left(x-x_{k}\right)\left(y-y_{l}\right)}{\left(x_{i}-x_{k}\right)\left(y_{j}-y_{l}\right)}\right)
$$

If experimental data is sparse, in other words, there is lack of some values $f\left(x_{i}, y_{j}\right)$, then, any expression $f\left(x_{i}, y_{j}\right)$ not evaluated is removed from the formula.

Now we consider the interpolation of derivative of bivariate function $f: \mathbb{R}^{2} \rightarrow \mathbb{R}$. The gradient vector of $f$ is:

$$
\nabla f=\left(\frac{\partial f}{\partial x}, \frac{\partial f}{\partial y}\right)
$$

Where $\frac{\partial f}{\partial x}$ and $\frac{\partial f}{\partial y}$ are partial derivatives with regard to $x$ and $y$, respectively. Note that the gradient $\nabla f$ is row vector. What we need to do is to take the interpolation to $\frac{\partial f}{\partial x}$ and $\frac{\partial f}{\partial y}$ individually. Let $\frac{\Delta f}{\Delta x}$ and $\frac{\Delta f}{\Delta y}$ be the approximations of $\frac{\partial f}{\partial x}$ and $\frac{\partial f}{\partial y}$, respectively, we have:

$$
\begin{aligned}
& \frac{\Delta f}{\Delta x}\left(x_{i}, y_{j}\right)=\frac{f\left(x_{i+1}, y_{j}\right)-f\left(x_{i}, y_{j}\right)}{x_{i+1}-x_{i}} \\
& \frac{\Delta f}{\Delta y}\left(x_{i}, y_{j}\right)=\frac{f\left(x_{i}, y_{j+1}\right)-f\left(x_{i}, y_{j}\right)}{y_{j+1}-y_{j}}
\end{aligned}
$$

Let $\frac{\Delta P}{\Delta x}$ and $\frac{\Delta P}{\Delta y}$ be the interpolation polynomials of $\frac{\Delta f}{\Delta x}$ and $\frac{\Delta f}{\Delta y}$, respectively, we have:

$$
\begin{aligned}
& \frac{\Delta P}{\Delta x}=\sum_{i=0}^{m-1} \sum_{j=0}^{n-1}(\left.\frac{\Delta f}{\Delta x}\left(x_{i}, y_{j}\right) P_{i j}\left(x_{i}, y_{j}\right)\right) \\
&= \sum_{i=0}^{m-1} \sum_{j=0}^{n-1}\left(\frac{f\left(x_{i+1}, y_{j}\right)-f\left(x_{i}, y_{j}\right)}{x_{i+1}-x_{i}} \prod_{\substack{k=0 \\
k \neq i}}^{m-1} \prod_{\substack{l=0 \\
l \neq j}}^{n-1} \frac{\left(x-x_{k}\right)\left(y-y_{l}\right)}{\left(x_{i}-x_{k}\right)\left(y_{j}-y_{l}\right)}\right) \\
& \frac{\Delta P}{\Delta y}=\sum_{i=0}^{m-1} \sum_{j=0}^{n-1}\left(\frac{\Delta f}{\Delta y}\left(x_{i}, y_{j}\right) P_{i j}\left(x_{i}, y_{j}\right)\right) \\
&=\sum_{i=0}^{m-1} \sum_{j=0}^{n-1}\left(\frac{f\left(x_{i}, y_{j+1}\right)-f\left(x_{i}, y_{j}\right)}{y_{j+1}-y_{j}} \prod_{\substack{k=0 \\
k \neq 1}}^{m-1} \prod_{\substack{l=0 \\
l \neq j}}^{n-1} \frac{\left(x-x_{k}\right)\left(y-y_{l}\right)}{\left(x_{i}-x_{k}\right)\left(y_{j}-y_{l}\right)}\right)
\end{aligned}
$$

Where $P_{i j}(x, y)$ is the indicator function as aforementioned. Please pay attention that any expression $f\left(x_{i+1}, y_{j}\right), f\left(x_{i}, y_{j}\right)$ or $f\left(x_{i}, y_{j+1}\right)$ not evaluated is removed from the formula. Now it is easy to determine the interpolation denoted $\Delta P$ of gradient vector $\nabla f$. 


$$
\Delta P=\left(\frac{\Delta P}{\Delta x}, \frac{\Delta P}{\Delta y}\right)
$$

Now we find out the interpolation of multivariate integral. Suppose $\left[x_{i}, x_{i+1}\right] \times\left[y_{i}, y_{i+1}\right]$ is a rectangle of given partition $x_{0} \leq x_{1} \leq \cdots \leq x_{m}$ and $y_{0} \leq y_{1} \leq \cdots \leq y_{n}$. Such rectangle and function $f$ form two triangular prisms denoted $T_{1}$ and $T_{2}$. Prism $T_{1}$ is created by five faces:

- $\quad$ Bottom triangular face $F_{11}=\left\{\left(x_{i}, y_{j}, 0\right),\left(x_{i}, y_{j+1}, 0\right),\left(x_{i+1}, y_{j+1}, 0\right)\right\}$.

- Top triangular face $F_{12}=\left\{\left(x_{i}, y_{j}, f\left(x_{i}, y_{j}\right),\left(x_{i+1}, y_{j+1}, f\left(x_{i+1}, y_{j+1}\right)\right),\left(x_{i}, y_{j+1}, f\left(x_{i}, y_{j+1}\right)\right)\right\}\right.$.

- Left-side trapezoidal face $F_{13}=\left\{\left(x_{i+1}, y_{j+1}, 0\right),\left(x_{i}, y_{j+1}, 0\right),\left(x_{i}, y_{j+1}, f\left(x_{i}, y_{j+1}\right)\right),\left(x_{i+1}\right.\right.$, $\left.y_{j+1}, f\left(x_{i+1}, y_{j+1}\right)\right\}$.

- Right-side trapezoidal face $F_{14}=\left\{\left(x_{i}, y_{j+1}, 0\right),\left(x_{i}, y_{j}, 0\right),\left(x_{i}, y_{j}, f\left(x_{i}, y_{j}\right)\right),\left(x_{i}, y_{j+1}, f\left(x_{i}\right.\right.\right.$, $\left.\left.y_{j+1}\right)\right\}$.

- Common rectangle face $F_{r}=\left\{\left(x_{i}, y_{j}, 0\right),\left(x_{i+1}, y_{j+1}, 0\right),\left(x_{i+1}, y_{j+1}, f\left(x_{i+1}, y_{j+1}\right)\right),\left(x_{i}, y_{j}\right.\right.$, $\left.\left.f\left(x_{i}, y_{j}\right)\right)\right\}$.

Similarly, prism $T_{2}$ is created by five faces:

- Bottom triangular face $F_{21}=\left\{\left(x_{i}, y_{j}, 0\right),\left(x_{i+1}, y_{j+1}, 0\right),\left(x_{i+1}, y_{j}, 0\right)\right\}$

- Top triangular face $F_{22}=\left\{\left(x_{i}, y_{j}, f\left(x_{i}, y_{j}\right)\right),\left(x_{i+1}, y_{j+1}, f\left(x_{i+1}, y_{j+1}\right),\left(x_{i+1}, y_{j}, f\left(x_{i+1}, y_{j}\right)\right)\right\}\right.$.

- Left-side trapezoidal face $F_{23}=\left\{\left(x_{i}, y_{j}, 0\right),\left(x_{i+1}, y_{j}, 0\right),\left(x_{i+1}, y_{j}, f\left(x_{i+1}, y_{j}\right),\left(x_{i}, y_{j}, f\left(x_{i}\right.\right.\right.\right.$, $\left.\left.\left.y_{j}\right)\right)\right\}$.

- $\quad$ Right-side trapezoidal face $F_{24}=\left\{\left(x_{i+1}, y_{j+1}, 0\right),\left(x_{i+1}, y_{j}, 0\right),\left(x_{i+1}, y_{j}, f\left(x_{i+1}, y_{j}\right),\left(x_{i+1}\right.\right.\right.$, $\left.\left.y_{j+1}, f\left(x_{i+1}, y_{j+1}\right)\right)\right\}$.

- Common rectangular face $F_{r}=\left\{\left(x_{i}, y_{j}, 0\right),\left(x_{i+1}, y_{j+1}, 0\right),\left(x_{i+1}, y_{j+1}, f\left(x_{i+1}, y_{j+1}\right)\right),\left(x_{i}, y_{j}\right.\right.$, $\left.\left.f\left(x_{i}, y_{j}\right)\right)\right\}$.

Both $T_{1}$ and $T_{2}$ have the same rectangle face $F_{r}$. Let $v\left(F_{13}\right), v\left(F_{14}\right), v\left(F_{23}\right)$ and $v\left(F_{24}\right)$ be areas of trapezoidal faces $F_{13}, F_{14}, F_{23}$ and $F_{24}$, respectively, we have:

$$
\begin{aligned}
& v\left(F_{13}\right)=\frac{1}{2}\left(f\left(x_{i}, y_{j+1}\right)+f\left(x_{i+1}, y_{j+1}\right)\right)\left(x_{i+1}-x_{i}\right) \\
& v\left(F_{14}\right)=\frac{1}{2}\left(f\left(x_{i}, y_{j}\right)+f\left(x_{i}, y_{j+1}\right)\right)\left(y_{j+1}-y_{j}\right) \\
& v\left(F_{23}\right)=\frac{1}{2}\left(f\left(x_{i+1}, y_{j}\right)+f\left(x_{i}, y_{j}\right)\right)\left(x_{i+1}-x_{i}\right) \\
& v\left(F_{24}\right)=\frac{1}{2}\left(f\left(x_{i+1}, y_{j}\right)+f\left(x_{i+1}, y_{j+1}\right)\right)\left(x_{i+1}-x_{i}\right)
\end{aligned}
$$

The volume of a triangular prism is the half of product of two areas of trapezoidal faces because the top and bottom faces of such prism are right triangles. Let $v\left(T_{1}\right)$ and $v\left(T_{2}\right)$ be volumes of prisms $T_{1}$ and $T_{2}$, respectively, we have:

$$
\begin{aligned}
& \begin{aligned}
v\left(T_{1}\right)=\frac{1}{2} * v( & \left.F_{13}\right) * v\left(F_{14}\right) \\
& =\frac{1}{8}\left(f\left(x_{i}, y_{j}\right)+f\left(x_{i}, y_{j+1}\right)\right)\left(f\left(x_{i}, y_{j+1}\right)+f\left(x_{i+1}, y_{j+1}\right)\right)\left(x_{i+1}-x_{i}\right)\left(y_{j+1}\right. \\
& \left.-y_{j}\right)
\end{aligned} \\
& \begin{aligned}
v\left(T_{2}\right)=\frac{1}{2} * v & \left(F_{23}\right) * v\left(F_{24}\right) \\
& =\frac{1}{8}\left(f\left(x_{i}, y_{j}\right)+f\left(x_{i+1}, y_{j}\right)\right)\left(f\left(x_{i+1}, y_{j}\right)+f\left(x_{i+1}, y_{j+1}\right)\right)\left(x_{i+1}-x_{i}\right)\left(y_{j+1}\right. \\
& \left.-y_{j}\right)
\end{aligned}
\end{aligned}
$$

Let $v\left(x_{i}, y_{j}\right)$ be the volume of polyhedron formed by the rectangle $\left[x_{i}, x_{i+1}\right] \mathrm{x}\left[y_{i}, y_{i+1}\right]$ and function $f$. It is easy to infer that the polyhedron is instituted of two prisms $T_{1}$ and $T_{2}$; in other words, the volume $v\left(x_{i}, y_{j}\right)$ is sum of $v\left(T_{1}\right)$ and $v\left(T_{2}\right)$ and so we have: 


$$
\begin{aligned}
v\left(x_{i}, y_{j}\right)=v( & \left.T_{1}\right)+v\left(T_{2}\right) \\
= & \frac{1}{8}\left[\left(f\left(x_{i}, y_{j}\right)+f\left(x_{i}, y_{j+1}\right)\right)\left(f\left(x_{i}, y_{j+1}\right)+f\left(x_{i+1}, y_{j+1}\right)\right)\right. \\
& \left.+\left(f\left(x_{i}, y_{j}\right)+f\left(x_{i+1}, y_{j}\right)\right)\left(f\left(x_{i+1}, y_{j}\right)+f\left(x_{i+1}, y_{j+1}\right)\right)\right]\left(x_{i+1}-x_{i}\right)\left(y_{j+1}\right. \\
& \left.-y_{j}\right)
\end{aligned}
$$

Please pay attention that any expression $f\left(x_{i+1}, y_{j}\right), f\left(x_{i}, y_{j}\right), f\left(x_{i}, y_{j+1}\right)$ or $f\left(x_{i+1}, y_{j+1}\right)$ not evaluated is removed from the formula. Suppose $s\left(x_{i}, y_{j}\right)$ be approximation of the integral of function $f$ on the $\left[x_{i}, x_{i+1}\right] \times\left[y_{i}, y_{i+1}\right]$, we recognize that $s\left(x_{i}, y_{j}\right)$ is sum of a block of volumes $v\left(x_{k}, y_{l}\right)$ where $k \leq i$ and $l \leq j$.

Where,

$$
s\left(x_{i}, y_{j}\right)=\sum_{k=0}^{i-1} \sum_{l=0}^{j-1} v\left(x_{k}, y_{l}\right)
$$

$$
s\left(x_{0}, y_{j}\right)=s\left(x_{i}, y_{0}\right)=0 \text { and } 1 \leq i \leq m \text { and } 1 \leq j \leq n
$$

Let $S(x, y)$ be the bivariate interpolation polynomial of integral of function $f$, we have:

$$
S(x, y)=\sum_{i=0}^{m} \sum_{j=0}^{n} s\left(x_{i}, y_{j}\right) P_{i j}(x, y)=\sum_{i=0}^{m} \sum_{j=0}^{n}\left(s\left(x_{i}, y_{j}\right) \prod_{\substack{k=0 \\ k \neq i \\ k \neq i}}^{m} \prod_{\substack{l=0 \\ l \neq j}}^{n} \frac{\left(x-x_{k}\right)\left(y-y_{l}\right)}{\left(x_{i}-x_{k}\right)\left(y_{j}-y_{l}\right)}\right)
$$

In similar to univariate case, the interpolation polynomial is deviated from the real integral of $f$ a constants $C$ with attention that the interpolation operation starts with the point $\left(x_{1}, y_{1}\right)$. The fact $s\left(x_{0}, y_{0}\right)=0$ implies that $S(x, y)$ is pulled back to the coordinate origin $(0,0,0)$ a constant $C$ according to $f(x, y)$-axis. Hence, $S(x, y)$ needs pushed away from the origin a distance $C$.

The constant $C$ is defined as volume of polyhedron formed by the rectangles $\left[x_{0}, x_{i}\right] \mathrm{x}\left[y_{0}\right.$, $\left.y_{j}\right]$ and function $f$.

$$
C=v\left(x_{0}, y_{0}\right)+\sum_{k=1}^{i-1} v\left(x_{k}, y_{0}\right)+\sum_{l=1}^{j-1} v\left(x_{0}, y_{l}\right)
$$

When $C$ is calculated, the adjusted interpolation polynomial denoted $A(x)$ are totally determined as following:

$$
A(x)=S(x, y)+C
$$

\section{Conclusion}

In general, there are two main aspects of the research:

- Proposing a method to interpolate polynomial of derivative and integral given a set of points. The ideology of such method is to make the discrete approximation of derivatives first, and then, to make interpolation on such approximations later. The method is opposite to normal way in which Lagrange interpolation is the most important procedure which is calculated first.

- Extending Lagrange interpolation to bivariate function $f$, in which the interpolation polynomial becomes two-variable function. When $f$ is multivariate function, the approach is keep intact but estimation formulas get much more complicated.

Derivative and integral interpolation with regard to two-variable function gets complicated because the main point of proposed method is to make approximations based on given experimental data. Derivative is translated as the speed of partial discrete variations. Integral is translated as a discrete sum of volumes of prisms. The purpose of these approximations is to eliminate the requirement that the derivative or integral of interpolation polynomial should 
be close to the derivative or integral of origin function when this requirement is the cause of inaccurate interpolation.

\section{Reference}

1. [Chapra, Canale 2010]. Steven C. Chapra and Raymond P. Canale. Numerical Methods for Engineers, 6th edition, ISBN: 978-0-07-340106-5, MHID: 0-07340106-4. Published by McGraw-Hill, Copyright (C) 2010 by The McGraw-Hill Companies, Inc.

2. [Ta 2014]. Duy-Phuong Ta. Lecture notes on Lagrange interpolation. Institute of Mathematics, Hanoi, Vietnam, 2014.

3. [Wikipedia 2014]. Lagrange polynomials. Last modified on 15 May 2014. Website link is http://en.wikipedia.org/wiki/Lagrange_polynomial. 